\title{
Anesthetic management including extracorporeal membrane oxygenation therapy of liver transplant recipient with life-threatening hypoxemia
} -a case report-

\author{
Chan Seon Yoo, Young Hee Shin, Justin Sangwook Ko, Mi Sook Gwak, and Gaab Soo Kim \\ Department of Anesthesiology and Pain Medicine, Samsung Medical Center, Sungkyunkwan University of Medicine, Seoul, Korea
}

We present a rare case of successful anesthetic management for a patient who had refractory hypoxia during liver transplantation (LT) with intraoperative veno-venous (VV) extracorporeal membrane oxygenation (ECMO) support. A 49 year-old female patient underwent living donor LT. After reperfusion of the grafted liver, graft congestion and massive oozing developed. Thus it was decided to reoperate with a temporary gauze packing. However, the patient's condition deteriorated with azotemia and severe hypoxemia. VV ECMO with continuous renal replacement therapy was started 24 hours before secondary LT and maintained during secondary LT. VV ECMO was weaned 32 hours after secondary LT. This case indicates that not only after the LT but also before and during LT, VV ECMO can be a treatment option for the patient with end-stage liver disease combined with respiratory failure when there is the possibility of lung recovery. (Korean J Anesthesiol 2013; 65: 151-157)

Key Words: Extracorporeal membrane oxygenation, Liver transplantation, Respiratory insufficiency.

Perioperative respiratory failure is relatively common in liver transplantation (LT), and it is very important for graft and recipient outcome [1,2]. Extracorporeal membrane oxygenation (ECMO) is an adaptation of conventional cardiopulmonary bypass technique that allows short-term support for potentially reversible acute respiratory or cardiac failure unresponsive to conventional therapeutic interventions $[3,4]$. In recent studies, veno-venous (VV) ECMO improved survival for adult patients with acute respiratory failure [3,5]. Despite a high incidence of preoperative respiratory failure, there are few reports of successful VV ECMO support during LT [6,7]. Patients who suffer respiratory failure while waiting for LT may be saved by this technique if it is appropriate for their condition. Here we report an experience of a successful anesthetic management in LT applying VV ECMO support.

Received: April 2, 2012. Revised: 1st, June 13, 2012; 2nd, July 24, 2012; 3rd, August 13, 2012. Accepted: August 20, 2012.

Corresponding author: Gaab Soo Kim, M.D., Department of Anesthesiology and Pain Medicine, Samsung Medical Center, Sungkyunkwan University of Medicine, 50, Ilwon-dong, Gangnam-gu, Seoul 135-710, Korea. Tel: 82-2-3410-0360, Fax: 82-2-3410-0361, E-mail: gskim@skku.edu (c) This is an open-access article distributed under the terms of the Creative Commons Attribution Non-Commercial License (http:// creativecommons.org/licenses/by-nc/3.0/), which permits unrestricted non-commercial use, distribution, and reproduction in any medium, provided the original work is properly cited. 


\section{Case Report}

A 49 year-old woman $(160.0 \mathrm{~cm}, 94.6 \mathrm{~kg})$ suffering from hepatocellular carcinoma with end-stage hepatitis B liver cirrhosis (model for end stage liver disease [MELD] score 14, Child-Pugh class B) presented for living donor LT. She had hypertension that was well controlled with medications. Her blood type was $\mathrm{A}, \mathrm{Rh}^{+}$. The $\mathrm{ABO}$ compatible donor was her 25 -year-old son $(174.5 \mathrm{~cm}, 86.2 \mathrm{~kg})$.

Anesthesia was induced with fentanyl $(100 \mu \mathrm{g})$, thiopental sodium (250 mg), and vecuronium ( $8 \mathrm{mg}$ ), and was maintained with oxygen, air, and desflurane. Neuromuscular block was maintained with atracurium. Hemodynamic monitoring included the right radial and femoral arterial, central and right femoral venous, pulmonary arterial, and capillary wedge pressures, and cardiac output measurements (Vigilance ${ }^{\mathrm{TM}}$ monitoring, Edwards Lifesciences, Irvine, CA, USA) through the right internal jugular vein.

The surgery proceeded by the piggyback technique, with preservation of caval flow. After the reperfusion of graft liver, right posterior sectionectomy was performed to improve the decreased right hepatic venous flow, a result of a relatively large size of graft liver (recipient original liver weight $=1,049 \mathrm{~g}$, graft volume $=1,500 \mathrm{ml}$, graft weight $=1,228 \mathrm{~g}$, graft-recipient weight ratio $=1.3 \%$, respectively) and morbid obesity (body mass index: $37.0 \mathrm{~kg} / \mathrm{m}^{2}$ ) of recipient. However, the graft blood flow did not improve, and intractable bleeding occurred at the resection site. Despite rapid volume resuscitation, hypovolemia

Table 1. Mechanical Ventilation and Arterial Blood Gas Analysis during Peri-ECMO Support

\begin{tabular}{|c|c|c|c|c|c|c|c|c|c|}
\hline & \multicolumn{4}{|c|}{ Ventilation } & \multirow[b]{2}{*}{$\mathrm{pH}$} & \multirow{2}{*}{$\begin{array}{c}\mathrm{PaCO}_{2} \\
(\mathrm{mmHg})\end{array}$} & \multirow{2}{*}{$\begin{array}{c}\mathrm{PaO}_{2} \\
(\mathrm{mmHg})\end{array}$} & \multirow{2}{*}{$\begin{array}{c}\mathrm{HCO}_{3}^{-} \\
(\mathrm{mmol} / \mathrm{L})\end{array}$} & \multirow{2}{*}{$\begin{array}{c}\mathrm{BE} \\
(\mathrm{mmol} / \mathrm{L})\end{array}$} \\
\hline & $\mathrm{FiO}_{2}$ & TV (ml) & $\begin{array}{c}\mathrm{RR} \\
\text { (breaths/min) }\end{array}$ & $\begin{array}{l}\text { PIP/PEEP } \\
\left(\mathrm{cmH}_{2} \mathrm{O}\right)\end{array}$ & & & & & \\
\hline Pre-CPR $\left(1^{\text {st }} \mathrm{LT}\right)$ & 0.46 & 560 & 10 & $26 / 5$ & 7.339 & 36.4 & 163.5 & 19.2 & -8.2 \\
\hline Post-CPR $\left(1^{\text {st }} \mathrm{LT}\right)$ & 0.70 & 580 & 12 & $29 / 5$ & 7.178 & 30.0 & 189.0 & 10.9 & -16.0 \\
\hline Before ECMO & 1.00 & 380 & 30 & $30 / 12$ & 7.353 & 38.1 & 56.9 & 20.7 & -4.4 \\
\hline After ECMO & 0.40 & 336 & 18 & $24 / 5$ & 7.448 & 26.5 & 57.3 & 17.9 & -4.7 \\
\hline Incision $\left(2^{\text {nd }} \mathrm{LT}\right)$ & 0.54 & 299 & 8 & $25 / 8$ & 7.325 & 33.6 & 117.2 & 17.1 & -8.1 \\
\hline Post-anhepatic, $1 \mathrm{~h}$ & 0.92 & 358 & 8 & $24 / 5$ & 7.316 & 28.9 & 366.8 & 14.4 & -10.6 \\
\hline Post-reperfusion, $5 \mathrm{~min}$ & 0.92 & 346 & 10 & $25 / 5$ & 7.256 & 35.6 & 250.2 & 15.5 & -10.7 \\
\hline Post-reperfusion, $3 \mathrm{~h}$ & 0.48 & 301 & 14 & $26 / 5$ & 7.279 & 40.4 & 84.4 & 18.5 & -7.7 \\
\hline Before weaning & 0.40 & 371 & 10 & $23 / 8$ & 7.386 & 35.7 & 115.4 & 21.6 & -2.4 \\
\hline Weaning, $1^{\text {st }}$ stage & 0.40 & 342 & 9 & $23 / 8$ & 7.390 & 42.8 & 81.1 & 25.3 & 0.3 \\
\hline Weaning, $2^{\text {nd }}$ stage & 0.60 & 417 & 14 & $25 / 5$ & 7.358 & 47.8 & 96.9 & 26.3 & 0.6 \\
\hline $48 \mathrm{~h}$ after ECMO weaned & 0.60 & 347 & 25 & $22 / 5$ & 7.402 & 39.6 & 74.4 & 24.1 & -0.6 \\
\hline
\end{tabular}

$\mathrm{FiO}_{2}$ : fraction of inspired oxygen, TV: tidal volume, RR: respiratory rate, PIP: peak inspiratory pressure, PEEP: positive end expiratory pressure, pH: hydrogen ion concentration, $\mathrm{PaCO}_{2}$ : partial pressure of carbon dioxide, $\mathrm{PaO}_{2}$ : partial pressure of oxygen, $\mathrm{HCO}_{3}^{-}$: bicarbonate ion, $\mathrm{BE}$ : base excess, CPR: cardiopulmonary resuscitation, LT: liver transplantation, ECMO: extra-corporeal membrane oxygenation.

Table 2. Coagulation Profiles during Peri-ECMO Support

\begin{tabular}{|c|c|c|c|c|c|}
\hline & Platelet $\left(1000 / \mathrm{mm}^{3}\right)$ & PT (INR) & $\mathrm{aPTT}(\mathrm{sec})$ & $\mathrm{ACT}(\mathrm{sec})$ & Fibrinogen (mg/dl) \\
\hline Pre-CPR $\left(1^{\text {st }} \mathrm{LT}\right)$ & 56 & 2.95 & 74.6 & - & 103 \\
\hline Post-CPR $\left(1^{\text {st }} L T\right)$ & 33 & $>10.00$ & $>300.0$ & - & 30 \\
\hline Before ECMO & 46 & 3.34 & 66.5 & 131 & - \\
\hline Preoperative & 54 & 2.16 & 57.3 & 135 & 74 \\
\hline Incision $\left(2^{\text {nd }} L T\right)$ & 59 & 3.02 & 71.4 & 135 & 90 \\
\hline Post-anhepatic, $1 \mathrm{~h}$ & 89 & 2.82 & 88.7 & - & 82 \\
\hline Post-reperfusion, $5 \mathrm{~m}$ & 75 & 2.74 & 89.5 & 150 & 84 \\
\hline Post-reperfusion, $1 \mathrm{~h}$ & 41 & 2.55 & 87.9 & 107 & 92 \\
\hline End of surgery & 38 & 2.10 & 65.0 & - & 121 \\
\hline Weaning, $1^{\text {st }}$ stage & 67 & 2.14 & 53.6 & 133 & - \\
\hline Weaning, $2^{\text {nd }}$ stage & 49 & 1.77 & 47.9 & 129 & - \\
\hline $48 \mathrm{~h}$ after ECMO weaned & 44 & 1.55 & 43.5 & - & 220 \\
\hline
\end{tabular}

PT: prothrombin time, INR: international normalized ratio, aPTT: activated partial thromboplastin time, ACT: activated clotting time, CPR: cardiopulmonary resuscitation, LT: liver transplantation, ECMO: extra-corporeal membrane oxygenation. 
was aggravated and then cardiac arrest occurred suddenly. Chest compression was started immediately by surgeon, and boluses of epinephrine $1 \mathrm{mg}$ were injected 4 times. Aggressive volume resuscitation was continued using a fluid management system (FMS 2000, Belmont Instrument Corporation, Billerica, MA, USA).

After 8 minutes of the cardiopulmonary resuscitation (CPR), spontaneous circulation was recovered with normal sinus rhythm. Arterial blood gas analysis (ABGA) and coagulation profiles at pre- and post-CPR are shown in Table 1 and Table 2. During CPR, serum potassium level was elevated $4.5 \mathrm{mmol} / \mathrm{L}$ to $5.1 \mathrm{mmol} / \mathrm{L}$ and serum calcium level decreased $0.62 \mathrm{mmol} / \mathrm{L}$ to $0.45 \mathrm{mmol} / \mathrm{L}$. Hematocrit level decreased 16 to $14 \%$. However, significant oozing continued despite surgical hemostasis, and the operator decided to stop surgery for intensive medical treatment and planned a second operation. Thus, after finishing the operation with gauze packing, the patient was transferred to surgical intensive care unit (ICU). During 1,270 minute operation, 33,255 $\mathrm{ml}$ of crystalloid, 1,300 $\mathrm{ml}$ of $5 \%$ albumin, 2,000 $\mathrm{ml}$ of colloid, 14 units of leukocyte depleted red blood cell, 6 units of leukocyte depleted platelet concentrate, $984 \mathrm{ml}$ of cell saver blood, 8 units of fresh frozen plasma, and 3 units of cryoprecipitate were infused. Urine output was $1,520 \mathrm{ml}$, and estimated blood loss was about $10,000 \mathrm{ml}$.

After the operation, the patient's condition deteriorated, with metabolic acidosis with $\mathrm{pH}<7.2$ and azotemia with creatinine level over $2.0 \mathrm{mg} / \mathrm{dl}$ due to ongoing graft failure and intractable bleeding despite aggressive management. Severe hypoxemia persisted despite a fraction of inspired oxygen $\left(\mathrm{FiO}_{2}\right)=1.0$ with mechanical ventilation set as $6 \mathrm{ml} / \mathrm{kg}$ of tidal volume (for ideal body weight), 30 breaths/minute, and increasing positive endexpiratory pressure (PEEP) of up to $10 \mathrm{cmH}_{2} \mathrm{O}$ (Table 1).

Chest radiography revealed pulmonary congestion (Fig. 1A) and echocardiography was performed to rule out cardiogenic pulmonary edema, but cardiac physiology, function, and structure were in normal without any abnormalities. As anuria developed 7 hours after the end of the operation, continuous renal replacement therapy (CRRT) was started via the right femoral vein following a continuous VV hemodiafiltration protocol. Brain tomography was obtained before CRRT, but there was no definite abnormal finding.

Because of the failure of conventional ventilatory support, and in the hope of liver retransplantation, the decision was made to initiate VV ECMO as a rescue device. Although she suffered hypovolemic arrest during first LT, post-operative echocardiography revealed normal heart function, physiology and structures. Therefore, the cardiologist in our ECMO team suggested that there was no need of cardiac support with ECMO. The existing central cannula and Swan-Ganz catheter was removed. A 28 French (Fr) drainage cannula $\left(\right.$ DLP $^{\circledR}$, Medtronic Inc., Minneapolis, MN, USA) was placed in the right femoral vein, and a 21 Fr Medtronic return cannula was placed in the right internal jugular vein. A CAPIOX emergent bypass system and circuit $\left(\mathrm{EBS}^{\circledR}\right.$, Terumo Inc., Tokyo, Japan) was primed with $470 \mathrm{ml}$ of normal saline. The CAPIOX EBS oxygenator kit contains $1.8 \mathrm{~m}^{2}$ of membrane lung surface. CRRT was continued through ECMO circuit (Fig. 2). VV ECMO circulation was maintained at $4.0-5.0 \mathrm{~L} / \mathrm{min}\left(2.0-2.5 \mathrm{~L} / \mathrm{min} / \mathrm{m}^{2}\right)$ with $5-6 \mathrm{~L} /$ min sweep gas flow at $100 \%$ oxygen. Heparin was not used because the patient had a severe bleeding tendency, and 500 IU of antithrombin was injected intravenously every 6 hour (Post-LT care protocol of our center). Activated clotting time (ACT) was measured every $5 \mathrm{~min}$ for the initial $15 \mathrm{~min}$ then 240 min thereafter. The ACT was maintained between 120-180 $s$ throughout. After ECMO treatment, the patient's condition improved to $95 \% \mathrm{SpO}_{2}$ with a $\mathrm{FiO}_{2}$ of $0.3-0.4$ at the ventilator and 1.0 at ECMO (Table 1).

About 24 hours after ECMO application, the patient was transferred to the operating room to receive deceased donor LT. The warm ischemic time of the donated liver was 41 minutes, and the cold ischemic time was 446 minutes.

Perioperative ABGA profiles and coagulation profiles are

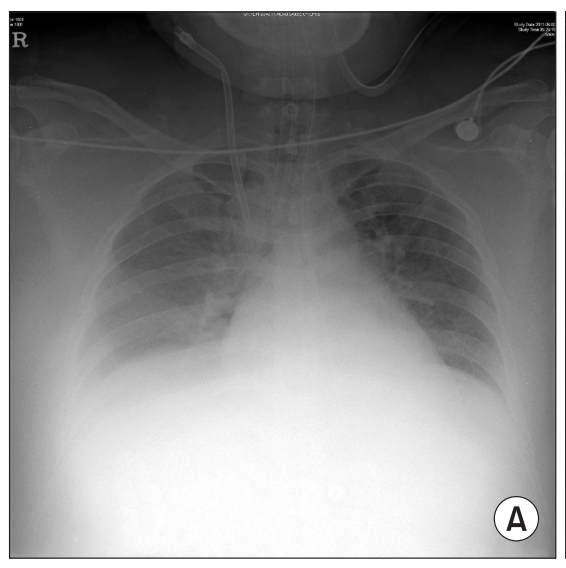

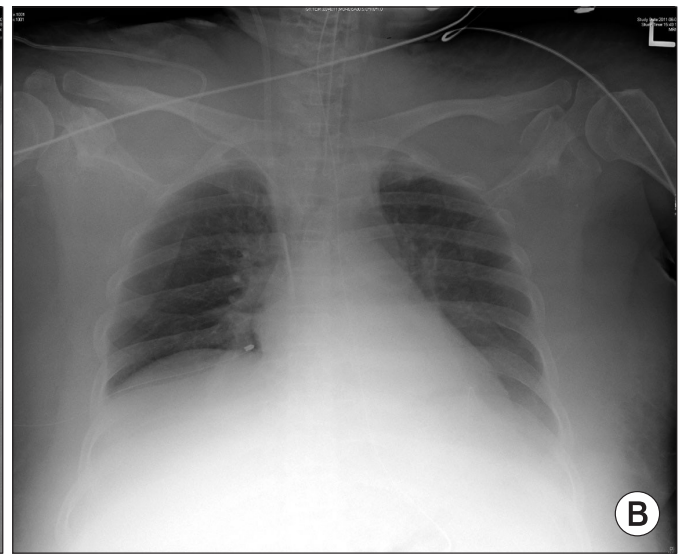

Fig. 1. Chest radiography before(A) and after (B) the ECMO support. Those showed improvement of punlmonary congestion after ECMO support. 


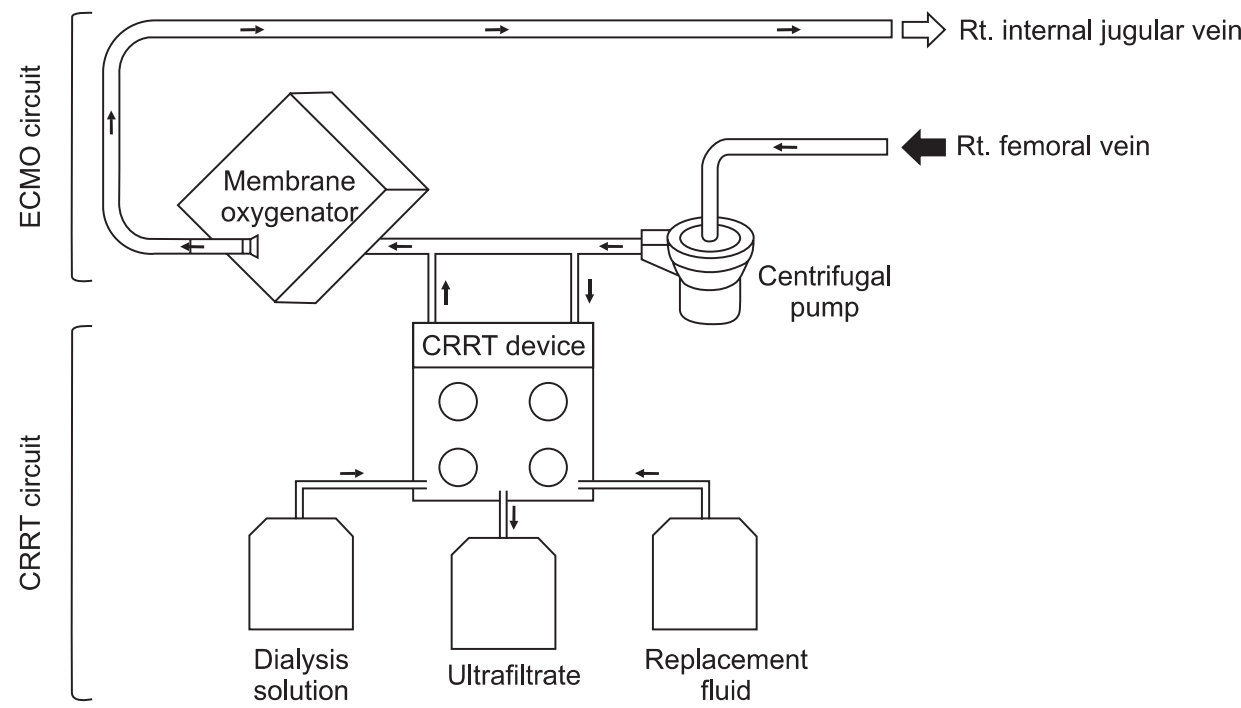

Fig. 2. Schematic diagram of veno-venous extracorporeal membrane oxygenation (ECMO) system combined with continuous renal replacement therapy (CRRT). shown in Table 1 and 2, respectively. The preoperative chest radiograph showed a sustained pulmonary congestion and bilateral pleural effusions. Her MELD score at secondary LT was 30 with ECMO and CRRT support. Considering her condition with a high risk of massive bleeding along with the planned ECMO application during the operation, we decided to discontinue CRRT during the operation and restart after the operation depending on her condition.

When the patient arrived at operating room, she was already intubated. The patient was monitored as during the previous operation except for femoral arterial blood pressure, pulmonary arterial pressure, and cardiac output monitoring, because there were no suitable cannulation site for these devices due to ECMO cannula and generalized edema. To maintain body temperature during anesthesia, core temperature was continuously monitored via mid-esophageal temperature probe. Administered fluid was heated by FMS 2000, and every peripheral vascular access was removed to prevent infusion of cold fluid. A warm blanket was spread on the operating table, and a humidifier (Hygroster, Covidien, Mansfield, MA, USA) was applied at the ventilatory circuit. The patient's limbs were covered with cotton roll and vinyl wrap and room temperature was maintained within 24.0 to $28.0^{\circ} \mathrm{C}$. Anesthetic management followed our hospital's LT protocol.

Her $\mathrm{SpO}_{2}$ was stable ranged from 97 to $100 \%$ with a $\mathrm{FiO}_{2}$ of 0.5 at the ventilator (pressure controlled ventilation; tidal volume of 3 to $4 \mathrm{ml} / \mathrm{kg}$ (about 5 to $6 \mathrm{ml} / \mathrm{kg}$ for ideal body weight), respiratory rate of 8 to 12 breaths/min, and PEEP of 5 to $10 \mathrm{cmH}_{2} \mathrm{O}$ ) and 1.0 at ECMO (Circuit flow of 4 to $5 \mathrm{~L}$ / min and gas sweep of 5 to $6 \mathrm{~L} / \mathrm{min}$ ). Her body temperature was maintained within the range of 35.8 to $36.1^{\circ} \mathrm{C}$.

Compartment syndrome was a concern during abdominal closure, so surgery was finished without suturing the abdominal wall. Total administered volume included crystalloid 7,400 $\mathrm{ml}$, colloid 2,530 ml, 5\% dextrose water $450 \mathrm{ml}$, cell saver autotransfusion $792 \mathrm{ml}$, leukocyte-depleted red blood cell 11 units, fresh frozen plasma 8 units, plateletpheresis 2 units, cryoprecipitate 8 units for 350 minutes. There was no severe oozing or acute massive bleeding on operation field. Hematocrit level was maintained over $24 \%$ during operation without rapid volume resuscitation. No hypotensive episode occurred below mean arterial pressure of $70 \mathrm{mmHg}$. Urine was not excreted during the entire operation.

After the surgery, sedation was maintained with continuous infusion of midazolam and fentanyl. CRRT was restarted and ECMO was continued. Her body temperature was maintained within the range of 35.3 to $37.0^{\circ} \mathrm{C}$ with forced-air warmer (Bair Hugger $^{\circledR}$, Augustine Medical, Eden Prairie, MN, USA), heated humidifier and circuit (MR850, Fisher and Paykel Healthcare, Auckland, New Zealand), and pre-warmed fluid. To check the return of consciousness, Glasgow coma scale was tested every 4 hours. Pupil size, light reflex, limb movement, and orientation were also checked to judge neurologic abnormalities. Pupil dilatation did not occur and light reflex was normal, but the other exams were meaningless during ECMO support because continuous sedation and muscular relaxation therapy with cisatracurium were sustained for the opened laparotomy wound.

After 64 hours following ECMO support, and 32 hours after secondary LT, arterial oxygen saturation (Table 1) and chest roentgenogram (Fig. 1B) were improved significantly. Based on sonography and improved coagulopathy (Table 2), the graft appeared functional. Hemodynamic stability was also achieved without high dose inotropic support $(0.7 \mathrm{mcg} / \mathrm{kg} / \mathrm{min}$ of norepinephrine was infused continuously before secondary LT, and tapered to $0.05 \mathrm{mcg} / \mathrm{kg} / \mathrm{min}$ at before ECMO weaning). Recovery from hepatic failure was the most important factor 
to decide weaning, because our patient's respiratory failure was closely related to hepatic failure. To initiate weaning, in the first stage, we reduced bypass flow from $4.0-5.0 \mathrm{~L} / \mathrm{min}$ to $3.0 \mathrm{~L} / \mathrm{min}$. And in the second stage, for 7 hours of close monitoring and ABGA at every 2 hours after start weaning, we reduced oxygen flow to ECMO to zero gradually, and $\mathrm{FiO}_{2}$ for ventilator was increased from 0.4 to 0.6 over an hour. Two hours after the second stage, ABGAs performed twice per hour showed appropriate oxygenation, then ECMO was removed from the patient. Table 1 shows ABGA profiles for both during and after ECMO weaning. Comparing just before and after the ECMO support, $\mathrm{PaO}_{2} / \mathrm{FiO}_{2}$ ratio improved from 56.9 to 117.2. The $\mathrm{PaCO}_{2}$ level was similar to before ECMO support, but ventilatory demand was significantly decreased. Subsequently, $\mathrm{PaO}_{2} / \mathrm{FiO}_{2}$ stabilized at $\mathrm{SpO}_{2}$ over $90 \%$ with $\mathrm{FiO}_{2}$ 0.6. The laparotomy wound closure was done 5 days after the surgery.

However, liver congestion was noted one day after the wound closure and hepatic drainage failed. Surgical decompression was performed, but stenosis at intrahepatic IVC developed. Although stenting for the stenosis was performed 5 days after the surgical decompression, graft failure was not improved. In addition, long-term use of high dose vasopressor led to both leg necrosis and further sepsis. After stopping fentanyl and cisatracurium infusion, the patient showed continuous eye opening for a half-day and low grade motor responses, but no further improvement of consciousness. The patient expired 24 days after the second LT due to multi-organ failure caused by combined graft failure and uncontrolled sepsis.

\section{Discussion}

Although respiratory failure occurs frequently in LT, LT with VV ECMO support has been rarely reported. To our knowledge, only one case of pediatric [6] patient and one case of adult [7] patient were reported in the English literature. But these two case reports did not describe the detail of perioperative ECMO use, only focusing the patients' status for considering the use of ECMO. As LT candidates with ECMO support tended to die waiting for LT usually, or most LT candidates did not fit the indication for ECMO support, LT using ECMO has been rarely reported.

However, the number of LT is growing and the recipient criteria are broadening these days. The need for ECMO treatment during LT may increase, posing anesthetic challenges. We experienced an anesthetic challenge in using VV ECMO during secondary LT in the patient with respiratory failure.

One anesthetic consideration in this case is a possibility of the expansion of operability in a patient with severe respiratory failure waiting for LT. Most patients who are waiting for LT have end-stage liver disease (ESLD) and they are at high risk of respiratory failure even before surgery [1]. In addition, LT is known to raise the risk of postoperative respiratory failure and acute pulmonary injury than heart or renal transplantations [2]. Arozullah et al. [8] performed a prospective cohort study to predict multifactorial risk index for postoperative respiratory failure associated with major non-cardiac surgery. They estimated that $37 \%$ of patients who received LT developed postoperative respiratory failure and were unable to extubate, and $29 \%$ of patients who developed post-operative respiratory failure needed re-intubation.

In making the decision to institute ECMO, several considerations must be weighed. Most importantly, one must consider the likelihood of organ recovery (reversibility of lung function in this case), that is, whether the patient would be weaned from ECMO treatment or not. In our patient, acute respiratory failure was mainly caused by liver graft dysfunction and persistent bleeding at the congested liver graft. It could be reversible by re-transplantation of the liver. We applied VV ECMO support to our patient, expecting successful weaning from ECMO management after second LT. There are many entry criteria for VV ECMO, and these differ among hospitals [9]. Our ECMO team do not use a single criterion, but the most frequently used is $\mathrm{PaO}_{2}<50 \mathrm{mmHg}$ for $>2$ hours at $\mathrm{FiO}_{2} 1.0$; PEEP $5 \mathrm{cmH}_{2} \mathrm{O}$ as fast entry criterion and $\mathrm{PaO}_{2}<50 \mathrm{mmHg}$ for $>12$ hours at $\mathrm{FiO}_{2} 0.6$; PEEP $5 \mathrm{cmH}_{2} \mathrm{O}$; maximal medical therapy over 48 hours as slow entry criterion [9]. Although her condition did not exactly meet these criteria, the situation called for aggressive treatment, that is, respiratory failure was worsening as time went on, and $\mathrm{PaO}_{2}$ was below $60 \mathrm{mmHg}$ during the last 26 hours before ECMO applied. This deteriorated condition was not expected to be resolved before secondary LT; thus we decided to apply ECMO.

ECMO provides blood oxygenation, temporary support for patients with severe respiratory or cardiac failure [3-5]. There are two types of ECMO: venous-arterial (VA) and VV ECMO [5]. VV ECMO supports isolated oxygenation failure, whereas VA ECMO provides hemodynamic and respiratory support. The VA ECMO changes the direction of blood flow, which may cause more complications (left ventricle distension, acute pulmonary hypertension, and even pulmonary hemorrhage) than VV ECMO. Because our patient had respiratory failure not responding to mechanical ventilation with an improved cardiac function after the intraoperative cardiopulmonary resuscitation, VV ECMO was instituted in this case. In VV ECMO, the patient's arterial oxygen saturation $\left(\mathrm{SaO}_{2}\right)$ depends on ECMO flow, native cardiac output, and oxygen consumption. With ECMO flows of 2-3 L/min $/ \mathrm{m}^{2}$, and assuming normal values of cardiac output and oxygen consumption, $\mathrm{SaO}_{2}$ values of $85-95 \%$ can be achieved in the absence of any pulmonary ventilation [10]. Lung protective ventilation is essential for lung recovery during ECMO [11]. We successfully provided lung protective 
ventilation during ECMO support (Table 1). There was only about a half -hour total of exceptions during secondary LT: ventricular tachycardia (VT) without hypotension appeared after IVC partial clamping to remove previously grafted liver (Table 1; Post-anhepatic, 1 hours), and around the reperfusion of graft liver (Table 1; Post-reperfusion, $5 \mathrm{~min}$ ). At these times, we modified $\mathrm{FiO}_{2}$ to 1.0 , to reduce risk of vital organ hypoperfusion. However, when VT and reperfusion syndrome was resolved after a few minutes without significant hypotension, we returned $\mathrm{FiO}_{2}$ to its prior state. The greatest anesthetic challenge in this case were bleeding tendencies and anticoagulation. During extracorporeal circulation, thrombosis may occur in the circuit and induce an embolism because of blood-surface interaction. As a result, anticoagulation with a continuous infusion of unfractionated heparin is mandatory. Before cannulation, 75$150 \mathrm{IU} / \mathrm{kg}$ of heparin needs to be administered to acquire activated clotting time (ACT) over 300 second. During ECMO support, heparin must be infused continuously to maintain ACT 180 to 240 second [11]. However, the target ACT should be decreased if the patients have a tendency towards bleeding.

In this case, conventional anticoagulation protocol was not suitable, because she had severe bleeding tendencies after the first LT. Bleeding tendency is frequently challenging in a patient who requires ECMO support, not only in LT patients. In such cases, systemic heparinization can be selectively applied, but there are no consistent guidelines or recommendations for the patient who has severe bleeding tendency. Muellenbach et al. [12] reported heparin-free ECMO support in traumatic brain injury patients. They did not use heparin even at the initiation of ECMO support to prevent worsening intracranial hemorrhage. If the bleeding tendency was treated effectively during ECMO support, they used low dose heparin to maintain aPTT within range of 40 to 50 seconds or 120 to 150 seconds on ACT. Compared to our case, bleeding tendency was improved but not completely resolved until ECMO was weaned (Table 2). Target aPTT or ACT was maintained without anticoagulation (Table 2). We use transfusion protocol (Table 3) for LT anesthesia of our

Table 3. Transfusion Protocol during Liver Transplantation



INR: international normalized ratio. center, and did not change the transfusion protocol during LT.

Weaning from ECMO should be carefully performed, reducing functional support of the ECMO gradually and evaluating the patient's tolerance of the reduced ECMO support conditions repeatedly. In other words, if adequate gas exchange is maintained on conventional ventilator support and the patient tolerates the reduced ECMO flow, a trial period without ECMO can be started [11]. With regards to VV ECMO, the actual ECMO flows do not need to be altered to assess native respiratory function-this is done by altering gas flow through the ECMO circuit. The patient may be weanable if gas exchange can be maintained with a low $\mathrm{F}_{\mathrm{i}} \mathrm{O}_{2}$ on the oxygenator and low fresh gas flow rates into the circuit provided that the respiratory rate and PEEP set on the ventilator are not too high $[13,14]$. Anticoagulation is also needed during ECMO weaning period. However, weaning from VV ECMO without heparinization can be possible to maintain ECMO flow in the patient with bleeding tendency [15]. In this case, the second stage of weaning proceeded with $3 \mathrm{~L} / \mathrm{min}$, relative low flow and no evidence of thromboembolic complications. Target ACT and aPTT level were maintained the same as ECMO maintenance (Table 2).

Although the duration of ECMO support was relatively short, pulmonary congestion and pulmonary effusion was improved on postoperative chest radiography and serial ABGAs revealed the patient's respiratory function was improved enough to wean from ECMO. Our ECMO team evaluated the ECMO function and thromboembolic complication periodically. Successful weaning from ECMO treatment was achieved 32 hours after second LT under lung protective strategies despite morbid obesity.

The patient could not achieve neurological recovery and expired 24 days after the second LT. However, these results might stem from hepatic encephalopathy and sepsis rather than the respiratory failure, because $\mathrm{PaO}_{2}$ level was not considered critically low (over $55 \mathrm{mmHg}$ even before the ECMO support). In addition, there were no marked neurological deficits and no abnormal finding on brain tomography even before ECMO treatment. So we achieved the 'primary goal' with this treatment that secured the operability for the second LT, but could not make the 'final goal' which means success of secondary LT and recovery of consciousness. However, this catastrophic course was associated with not the respiratory dysfunction but the hepatic failure and sepsis resulted from surgical complication. Thus, we conclude that this result does not alter the significance of ECMO treatment.

In conclusion, this case indicates that VV ECMO can be a treatment option for the patient with end-stage liver disease combined with respiratory failure, who has a possibility of lung recovery, both before and during LT, but further case experiences are necessary to decide the appropriate anticoagulation. 


\section{References}

1. Hemprich U, Papadakos PJ, Lachmann B. Respiratory failure and hypoxemia in the cirrhotic patient including hepatopulmonary syndrome. Curr Opin Anaesthesiol 2010; 23: 133-8.

2. Huang CT, Lin HC, Chang SC, Lee WC. Pre-operative risk factors predict post-operative respiratory failure after liver transplantation. PLoS One 2011; 6: e22689.

3. Bastin AJ, Firmin R. Extracorporeal membrane oxygenation for severe acute respiratory failure in adults: NICE guidance. Heart 2011; 97: 1701-3.

4. Chauhan S, Subin S. Extracorporeal membrane oxygenation, an anesthesiologist's perspective: physiology and principles. Part 1. Ann Card Anaesth 2011; 14: 218-29.

5. Lindstrom SJ, Pellegrino VA, Butt WW. Extracorporeal membrane oxygenation. Med J Aust 2009; 191: 178-82.

6. Landsman IS, Karsanac CJ. Case report: pediatric liver retransplantation on an extracorporeal membrane oxygenation-dependent child. Anesth Analg 2010; 111: 1275-8.

7. Monsel A, Mal H, Brisson H, Luo R, Eyraud D, Vezinet C, et al. Extracorporeal membrane oxygenation as a bridge to liver transplantation for acute respiratory distress syndrome-induced life-threatening hypoxaemia aggravated by hepatopulmonary syndrome. Crit Care 2011; 15: R234.

8. Arozullah AM, Daley J, Henderson WG, Khuri SF. Multifactorial risk index for predicting postoperative respiratory failure in men after major noncardiac surgery. The National Veterans Administration Surgical Quality Improvement Program. Ann Surg 2000; $232: 242-53$.

9. Lewandowski K. Extracorporeal membrane oxygenation for severe acute respiratory failure. Crit Care 2000; 4: 156-68.

10. Smith IJ, Sidebotham DA, McGeorge AD, Dorman EB, Wilsher ML, Kolbe J. Use of extracorporeal membrane oxygenation during resection of tracheal papillomatosis. Anesthesiology 2009; 110: 427-9.

11. Chauhan S, Subin S. Extracorporeal membrane oxygenation--an anaesthesiologist's perspective--Part II: clinical and technical consideration. Ann Card Anaesth 2012; 15: 69-82.

12. Muellenbach RM, Kredel M, Kunze E, Kranke P, Kuestermann J, Brack A, et al. Prolonged heparin-free extracorporeal membrane oxygenation in multiple injured acute respiratory distress syndrome patients with traumatic brain injury. J Trauma Acute Care Surg 2012; 72: 1444-7.

13. Hartwig MG, Appel JZ 3rd, Cantu E 3rd, Simsir S, Lin SS, Hsieh CC, et al. Improved results treating lung allograft failure with venovenous extracorporeal membrane oxygenation. Ann Thorac Surg 2005; 80: 1872-9.

14. Marasco SF, Lukas G, McDonald M, McMillan J, Ihle B. Review of ECMO (extra corporeal membrane oxygenation) support in critically ill adult patients. Heart Lung Circ 2008; 17 Suppl 4: S41-7.

15. Lappa A, Donfrancesco S, Contento C, Vitalini E, Pisani P, Menichetti A, et al. Weaning from venovenous extracorporeal membrane oxygenation without anticoagulation: is it possible? Ann Thorac Surg 2012; 94: e1-3. 